Nouvelles perspectives en sciences sociales

Revue internationale de systémique complexe et d'études relationnelles

\title{
Chantiers de rénovation urbaine : nouveaux terrains pour la recherche en sciences sociales
}

\section{Nicolas Lamic}

Volume 7, numéro 2, mai 2012

Sur le thème de la modélisation

URI : https://id.erudit.org/iderudit/1013058ar

DOI : https://doi.org/10.7202/1013058ar

Aller au sommaire du numéro

Éditeur(s)

Prise de parole

ISSN

1712-8307 (imprimé)

1918-7475 (numérique)

Découvrir la revue

Citer cet article

Lamic, N. (2012). Chantiers de rénovation urbaine : nouveaux terrains pour la recherche en sciences sociales. Nouvelles perspectives en sciences sociales, 7(2), 173-205. https://doi.org/10.7202/1013058ar
Résumé de l'article

Le matériau utilisé pour la rédaction de cet article est issu de l'une des interventions psychosociologiques que nous avons réalisées dans le cadre de projets de rénovation urbaine en Martinique au cours des années 2006-2008. La problématique que nous soulevons est celle du risque de dérive technocratique qui menace l'action publique dès lors qu'elle ne se donne pas les moyens d'associer les individus et les groupes dans la détermination des objectifs qu'elle poursuit. C'est ainsi que nous montrons comment l'analyse des présupposés qui organisent l'action publique dans ces sociétés postcoloniales permet d'illustrer la violence exercée contre certaines catégories de la population martiniquaise. 


\title{
Chantiers de rénovation urbaine : nouveaux terrains pour la recherche en sciences sociales
}

\author{
Nicolas Lamic \\ Université des Antilles et de la Guyane \\ Campus de Schœlcher
}

e matériau utilisé pour cet article est issu de l'une des interle cadre de projets de rénovation urbaine en Martinique au cours des années 2006-2008. Comme d'autres, les sociétés antillaises ne semblent pas pouvoir échapper à la montée de l'impératif participatif dans les politiques publiques. La problématique que soulèvent ces opérations est donc celle des modalités d'insertion des dispositifs de concertation dans un ensemble dont l'histoire du peuplement, l'esclavage et le système de plantation, attestent du caractère d'extrême contrainte à l'origine de l'émergence de la société martiniquaise. Ce point commun qu'elle partage avec d'autres a permis aux anthropologues de l'étudier comme participant à ce vaste ensemble socioculturel qu'ils définissent comme le «monde noir des Amériques ». Pour Denys Cuche :

Le terme d'Amériques noires désigne l'ensemble des régions du Nouveau Monde qui ont été culturellement marquées par la présence massive d'esclaves africains et de leurs descendants. Que ce soit en Amérique du Nord, en Amérique centrale, en Amérique du Sud ou dans l'archipel des Caraïbes, un même héritage historique, l'esclavage et le système de 
plantation, a abouti à une certaine unité, par-delà leur diversité, des Amériques noires, tant sur le plan social que culturel ${ }^{1}$.

Cependant, il n'a pas échappé à Richard Price, cité par Christine Chivallon ${ }^{2}$, que l'unité de cette région semble aussi tenir à un autre caractère, celui d'une vitalité culturelle visible dans le domaine de la religion, de la musique et de la langue et dont ont su faire preuve ces communautés noires, dans leur nouvel environnement, en réponse à la discrimination raciale. Si bien que, pour rendre intelligible cette émergence, s'est constitué, du côté anglo-saxon, un courant de recherche en sciences sociales, les postcolonial studies $^{3}$, qui s'efforce de saisir la singularité des formes de socialité à travers lesquelles ces sociétés construisent leur unité.

C'est dans ce contexte qu'il nous est apparu que la problématique qui est la nôtre pourrait difficilement trouver une solution satisfaisante sous l'angle d'une ingénierie de la participation ${ }^{4}$. Car il s'agirait de penser que la problématique de l'intervention est soluble dans une pratique inscrite dans l'importation dans ces sociétés des modèles et des pratiques qui ont fait leur preuve ailleurs ${ }^{5}$. C'est contre cette tendance que semble nous mettre en garde Loïc Blondiaux. En donnant pour exemple les expériences conduites en Angleterre, par le New Labour, il souligne la façon dont la participation peut prendre les traits d'un instrument de dépolitisation au service d'un projet néolibéral'.

C'est ainsi qu'il apparaît que le fait, pour ces pratiques, de se

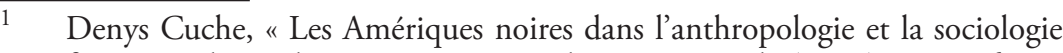
françaises depuis les Amériques noires de Roger Bastide (1967) ", Bastidiana, $\mathrm{n}^{\text {os }} 13-14,1996$, p. 119.

2 Christine Chivallon, La Diaspora noire des Amériques, Paris, CNRS, 2004, p. 14 .

3 La publication du livre de Paul Gilroy, L'Atlantique noir, Cahors, Kargo, 2003, a joué un rôle décisif dans l'émergence de ce courant de recherche.

4 Magali Nonjon, "Les Professionnels de la participation : savoir gérer son image militante ", Politix, vol. 2, n 70, 2005, p. 89-112.

5 Voir l'article de Marie-Hélène Bacqué et al, «La démocratie urbaine face au néo-libéralisme ", Mouvements, vol. 3, nºs 39-40, 2005, p. 121-131, pour une typologie des modèles participatifs.

6 Loïc Blondiaux, Le Nouvel Esprit de la démocratie, Paris, Seuil et la République des idées, 2008, p. 19. 
développer sous couvert d'un ensemble de dispositions légales ne saurait conduire à ignorer les autres registres (imaginaire, politique, économique...) à partir desquels elles s'élaborent ${ }^{7}$. JeanClaude Hélin, analysant le contenu de l'article du code de l'urbanisme sur l'obligation de la concertation, souligne que cette loi institue plus une simple obligation de procédure qu'une véritable procédure participative. Il note par ailleurs que c'est "l'absence de garanties pour le public qui constitue le maillon le plus faible de cette obligation procédurale ${ }^{8}$ ».

Cependant, et malgré toutes les difficultés qui semblent faire obstacle à la mise en ouuvre d'une véritable procédure de concertation citoyenne, nous sommes prêts à reconnaître avec d'autres auteurs que cette profusion d'initiatives traduit bien l'émergence d'un «nouvel esprit» de l'action publique moderne'. C'est dans ce sens que nous estimons que la visée première de l'intervention doit être celle qui consiste à faire du dispositif un lieu dans lequel les conflits vont pouvoir s'inscrire et si possible trouver une réponse dans les décisions publiques prises au terme de processus participatifs jouissant d'un taux d'adhésion collective significatif. Dans ce cadre, le travail d'analyse, en tant que démarche d'élucidation soutenue par des valeurs d'ordre démocratique, parait constituer une dimension nécessaire à toute procédure ayant " recours à la participation du citoyen ordinaire à la décision publique $^{10} »$.

Mais tout ceci ne va pas sans susciter quelques questions. Et parmi celles que l'on pourrait formuler, il y en a une qui concerne la capacité pour les personnes et les groupes de supporter les possibilités de confrontations directes que suppose le fonctionnement de tels dispositifs très localisés. Ne risque-t-on pas ainsi

Cécile Blatrix, "La Démocratie participative en représentation», Sociétés contemporaines, $\mathrm{n}^{\circ}$ 74, 2009, p. 97-119.

8 Jean-Claude Hélin, « La Concertation de l'article L. 300-2 du code de l'urbanisme ", Les cahiers du GRIDAUH, 2006, p. 213.

9 Voir les travaux de Patrick Savidan, "Démocratie participative et conflit ", Revue de métaphysique et de morale, $\mathrm{n}^{\circ}$ 58, 2008, p. 177-189, et ceux de Loïc Blondiaux et Yves Sintomer, "L'Impératif délibératif ", Rue Descartes, vol. 1, $n^{\circ} 63,2009$, p. 28-38.

10 Cécile Blatrix, op. cit., p. 97. 
de voir des éléments plus structuraux des conflits sociaux être écartés des controverses, faute de trouver les médiations nécessaires pour s'exprimer?

Notre hypothèse concernant le fonctionnement de ce type de dispositif dans le cadre d'une société comme la Martinique est la suivante : l'engagement dans la voie de l'assimilation politique, ayant indéniablement favorisé la modernisation de l'économie, a rendu plus complexe la structure sociale héritée des phases antérieures et créé une diversification d'intérêts. Cependant, la dimension autoritaire et rigide de l'intégration de la société martiniquaise à la nation - réclamant en contrepartie l'oblitération de ce qui est constitutif de différences et bien sûr d'inégalités, surtout raciales -, de même que la sur-représentation d'une élite de couleur - très imprégnée des idées et des valeurs héritées du colonialisme -, parmi le personnel politique, tout comme la déstructuration de la paysannerie ${ }^{11}$ - empêchée de peser dans le débat sur l'inégale répartition du patrimoine foncier -, constituent des tendances contraires limitant considérablement l'impact de ce phénomène dans l'émergence de conflits qui illustreraient la diversification sociale. Ainsi ce dont témoigneraient les obstacles auxquels se heurte l'expérimentation de dispositifs participatifs dans le champ des politiques publiques, c'est le refus communautaire de maîtriser les instituants de la vie sociale propres à la création d'une arène politique en rapport avec l'existence d'une structure de classe achevée.

Les quartiers dans lesquels nous sommes intervenu et d'où nous puisons la matière de cet article se situent sur le territoire urbanisé de Fort-de-France et de Schoelcher, couple qui forme

11 Cette hypothèse prend appui sur les travaux de Christine Chivallon, Espace et identité à la Martinique, Paysannerie des mornes et reconquête collective 1840-1960, Paris, CNRS, 2004, qui montrent comment s'est constituée une paysannerie martiniquaise aux lendemains de 1848 et de ceux de Raymond Massé, La Fin des plantations? Evolution des formes de soumission du travail dans deux sociétés néo-coloniales: Martinique et Guadeloupe, Montréal, Publication du Centre de recherche Caraïbes, Université de Montréal, 1980, qui font apparaître de quelle façon la départementalisation a entraîné une déstructurations de l'environnement du monde agricole dans les Antilles françaises. 
la partie la plus densément construite de l'agglomération foyalaise ${ }^{12}$. Le peuplement de ces quartiers a son origine dans l'appropriation, hors de toute légalité, de terrains inoccupés par des personnes démunies. Ce phénomène largement répandu devrait interroger, selon nous, l'institution sur la question de l'inégalité de la répartition du patrimoine foncier entre les différents groupes sociaux constitutifs de la société martiniquaise. Or, nous avons constaté que cette question, mêlant des significations en rapport avec l'histoire de la formation de ces sociétés, ne trouvait aucun écho dans le traitement du volet social de ces opérations. Dans ce domaine, les décideurs, plus soucieux de prévenir les risques environnementaux en rapport avec la qualité du bâti (risque sismique, problème d'insalubrité...), semblent reléguer à l'arrière-plan de leurs préoccupations les liens de causalité que l'on peut établir entre l'urbain et les avatars de la construction du lien social (violence urbaine, précarité et ségrégation sociale...). D'où l'idée que nous avons eue de montrer comment l'intervention, en maintenant une perspective scientifique suffisamment ouverte, peut contribuer à faire progresser l'analyse de phénomènes sociaux qui, jusqu'à présent, ont davantage été pris comme objets littéraires ${ }^{13}$ et moins considérés par des chercheurs en sciences humaines et sociales comme des objets dignes d'un intérêt scientifique ${ }^{14}$.

Il nous est apparu que les commanditaires, en l'occurrence les décideurs politiques locaux, alors même qu'ils pouvaient faire appel à des intervenants censés les aider à travailler sur la cohésion sociale dans la conduite de ces opérations, avaient plutôt tendance à établir le dispositif d'intervention au regard du cadre légal établi par la loi française concernant le droit de propriété. Du

12 Ce territoire regroupe un quart de la population martiniquaise. Ce phénomène de macrocéphalie urbaine, en nette régression par rapport aux années 1960, n'atteste pas moins de l'influence de la ville/capitale sur la vie insulaire.

13 Voir le roman Texaco de Patrick Chamoiseau (Gallimard, 1992) ou encore ceux de Raphaël Confiant qui prennent pour cadre, la ville.

14 Dans ce contexte, les travaux de Denis Martouzet font figure d'exception. (Denis Martouzet, « Le Rapport affectif à la ville, conséquences urbaines et spatiales. Le cas de Fort-de-France ", Annales de Géographie, vol. 111, nº 623, 2002, p. 73-85). 
coup, sur le plan de la représentation, ces populations démunies apparaissaient davantage comme des "hors-la-loi " que comme des personnes que l'on pourrait considérer comme des «victimes " d'un fonctionnement social établi en leur défaveur. Le terme de "marchands de sommeil » était utilisé, par exemple, pour désigner les propriétaires non-occupants qui louaient, dans ces quartiers périphériques du centre-ville, les logements qu'ils avaient auparavant occupés. Il ne semblait donc pas pouvoir venir à l'esprit de nos commanditaires que ces personnes, issues des rangs mêmes de cette population défavorisée, avaient su mettre en œuvre des moyens pour s'élever au dessus de leur condition, et cela en dépit des obstacles qu'elles avaient pu rencontrer au départ. En ce sens, ces discours stigmatisants visaient à dire comment elles devaient être traitées dans le dispositif de l'intervention, à leur assigner une place dans la hiérarchie sociale. À première vue, ils ne paraissaient pas traduire une volonté d'établir le dispositif sur le modèle des expériences de démocratie participative dont Porto Alegre représente le cas concrets le plus étudié ${ }^{15}$.

Cependant, et dans la mesure où le contenu de ces discours ne se situait pas non plus en référence à des dimensions d'ordre ethnique ou culturel en lien avec l'identité des personnes, il ne nous semblait pas possible de saisir la dynamique de ces rapports à l'aide d'une grille de lecture élaborée à partir des résultats des recherches anthropologiques conduites dans ces sociétés. Celles-ci, comme on le sait, ont longtemps cru trouver un modèle explicatif dans le type de rapport social institué dans le cadre de la plantation. Dans le système fondé sur le "duo maître/esclave ", où la couleur seule déterminait la place que l'individu occupait dans le rapport de production, tout espoir pour les Noirs en tant que groupe culturel d'améliorer leur condition était en effet exclu. Or, ce que nous permettait de voir notre positionnement dans le cadre de l'intervention, c'était que l'implantation récente

15 Le livre écrit par Marion Gret et Yves Sintomer, Porto Alegre, l'espoir d'une autre démocratie, Paris, La Découverte, 2002, présente dans le détail le dispositif participatif mis en place par les autorités municipales de la ville de Porto Alegre au Brésil pour impliquer la population dans la gestion des affaires publiques. 
d'une population d'origine rurale dans le milieu urbain obligeait à prendre en compte dans l'analyse le rôle de la ville, en tant que forme sociale spécifique, renouvelant ainsi le cadre de la recherche en sciences sociales s'intéressant à l'évolution des sociétés postcoloniales françaises.

\section{Arguments théoriques en faveur d'un changement de paradigme}

C'est ainsi que nous avons pris conscience que ces discours se situaient en référence à une représentation des rapports sociaux analysables selon une perspective théorique puisant davantage ses références dans la sociologie. Roger Bastide s'était déjà rendu à cette évidence, en se penchant sur l'étude de ces sociétés dans Les Amériques noires, puisqu'il déclarait que : "le Noir a, pour monter dans l'échelle sociale, assimilé complètement les valeurs des Blancs [...] il y a sans doute des différences d'épiderme - mais pas de différence de genres de vie. Ces sociétés relèvent de la sociologie, non de l'anthropologie culturelle ${ }^{16}{ }^{\prime}$. Cependant, il paraît difficile de pouvoir concilier les propos de Bastide avec ceux de J. Smeralda-Amon qui considère que la présence d'un groupe de blancs créoles sur ces territoires constitue un obstacle redoutable à toute analyse du lien social. Pour elle : «l'isolat qu'il constitue complique tout effort de conceptualisation du lien social qui régit la vie des groupes à la Martinique, que cet effort soit entrepris dans une perspective horizontale, verticale ou croisée ${ }^{17}$ ". Selon J. Smeralda-Amon, c'est la présence de ce groupe qui a inspiré toute une tradition anthropologique mettant l'accent sur la "survivance » de traits culturels propres à cette société. Cependant, les propos de Bastide sur les stratégies que les Noirs, pris en tant que groupe culturel, mettent en ouvre dans les cadres de jeux plus ouverts quautorise le fonctionnement des sociétés démocratiques, laissent apparaître des modes de sujétion autrement plus subtils que ceux qui feraient reposer l'orientation des conduites et des rapports uniquement sur des fondements d'ordres eth-

\footnotetext{
16 Roger Bastide, Les Amériques noires, Paris, L'Harmattan, 1996, p. 50.

17 Juliette Smeralda-Amon, La Racisation des relations intergroupes ou la problématique de la couleur, Paris, L'Harmattan, 2002, p. 241.
} 
nique ou culturel. "Par un paradoxe apparent, mais qui cache une logique certaine, c'est dans le régime concurrentiel que le nègre, pour acquérir les mêmes droits, économiques, politiques ou sociaux que le Blanc, abandonne son héritage africain pour s'occidentaliser ${ }^{18}$ ". Car, il ne semble pas échapper à Bastide que dans ces sociétés segmentées, les groupes sociaux dominés sont confrontés à diverses formes de violence. C'est ainsi que Raymond Massé identifie une forme de violence insidieuse dans la mesure où elle apparaît être de type structurel. En s'appuyant sur les travaux de Paul Farmer, il la définit comme toute "violence exercée systématiquement, c'est-à-dire indirectement, par tous ceux qui appartiennent à un certain ordre social ${ }^{19} \%$. Il s'agit, ajoute-t-il, d'une violence (ex : violence économique, symbolique, discrimination raciale) dont les impacts sont tout aussi réels sur l'intégrité physique et mentale des populations qui en sont victimes que le seraient d'autres formes de violence exercées plus directement. Et, pour être plus précis, il écrit : «Aux Antilles françaises, les rapports sociaux de couleur, la dépendance politique et économique face à la Métropole (et à l'Union européenne) et le chômage structurel constituent des exemples de telles violences structurelles ${ }^{20}$ ».

Pour autant, Massé n'oublie pas de souligner que l'élévation du niveau de vie des sociétés françaises d'Amérique semble constituer le pendant de la politique économique postcoloniale orchestrée depuis quelques décennies dans ces territoires. De la même manière, il note que l'accession à la citoyenneté française, en octroyant des droits civiques aux individus, et l'augmentation des mariages mixtes participent à protéger contre les discriminations. Alors, s'il convient de ne pas minorer ces aspects dans l'analyse, on peut quand même s'interroger sur la capacité de ces solidarités structurelles de participer mécaniquement à la construction de cadres de vie fondés sur le sentiment d'une " identité communautaire partagée " et d'une "solidarité

$\overline{18}$ Roger Bastide, op. cit., p. 218.

19 Raymond Massé, Détresse créole, Québec, PUL, 2008, p. 21.

20 Ibid., p. 21. 
culturelle ".

À partir de l'instant où l'on conçoit l'intérêt d'intégrer la dimension institutionnelle ${ }^{21}$ dans l'analyse, il apparaît, à l'époque où ces anciennes colonies accèdent au statut de département français d'outre-mer et leurs populations à celui de citoyens de la République, que l'intervention de l'Etat sur ces territoires se confine en un jeu subtil, " mêlant action sociale, action psychologique et répression ${ }^{22}$ ", dans l'objectif avoué d'interdire toute contestation de la politique gouvernementale. C'est l'époque du BUMIDOM (Bureau pour l'immigration de la population originaire des DOM dont les missions ont été fixées par un arrêté de 1963), de l'ordonnance Debré de 1960 autorisant les pouvoirs publics français à procéder à l'exil forcé en métropole des fonctionnaires de l'outre-mer troublant l'ordre public. C'est ainsi que la ville, en tant que lieu de concentration, représente pour ces opérations un espace facilité de contrôle, de surveillance et d'encadrement de la population.

Aussi n'est-il pas fortuit de noter que dans un livre écrit en 2007, Robert Castel s'interroge encore sur la possibilité qu'il y ait toujours à l'œuvre dans la société française : « une conception rigide et autoritaire de l'appartenance nationale qui exige comme idéal, à la manière dont l'idéologie coloniale pensait l'assimilation, que l'on renonce à toute spécificité renvoyant à une autre origine pour se couler dans le moule de la République ${ }^{23}$ ". Ce qui nous incite à concevoir l'hypothèse d'une diffusion de cette idéologie bien au-delà des limites du champ administratif et réglementaire et donc à chercher à saisir ses effets dans le réel à travers les discours et les actes que des personnes et des groupes

$21 \quad$ Cette dimension de l'analyse se situe en référence aux travaux des auteurs qui se réclament du courant de l'analyse institutionnelle, et plus particulièrement à ceux développés par Rémi Hess dans son ouvrage intitulé Centre et périphérie et dans lequel le modèle centre/périphérie fonctionne comme une grille d'analyse du pouvoir; Rémi Hess, Centre et périphérie, Paris, Anthropos, 2001, p. 139.

22 Mathieu Rigouste, L’Ennemi intérieur, La généalogie coloniale et militaire de l'ordre sécuritaire dans la France contemporaine, Paris, La Découverte, 2009.

23 Robert Castel, La Discrimination négative. Citoyens ou indigènes?, Paris, Seuil, 2007, p. 93-94. 
peuvent mettre en ouvre pour prendre part à la construction de leur cadre de vie.

Ces éléments de réflexion théorique font partie de la démarche clinique qui a été la nôtre dans le cadre de la conduite d'un chantier de rénovation urbaine sur lequel nous sommes intervenu en tant que psychosociologue. Le choix de cette approche semble s'imposer dès lors que l'intervenant est en mesure de concevoir que l'option qui consiste à vouloir impliquer les acteurs dans les objectifs et les processus de changement ne peut constituer une voie pour résoudre des difficultés que si ces derniers ont les moyens de s'engager de façon autonome dans la situation. C'est ainsi que l'on peut définir l'approche clinique comme : "une approche qui privilégie la construction du sens et l'accroissement, par les acteurs sociaux, de la connaissance des processus dans lesquels ils sont pris et de la façon dont ils en sont partie prenante ${ }^{24}$ ". Cette tentative d'articulation de différents modèles théoriques répond donc au souci de faire apparaître, de façon plus formelle, le risque de dérive technocratique qui menace l'action publique dès lors qu'elle ne se donne pas les moyens d'associer les individus et les groupes dans la détermination des objectifs qu'elle poursuit ${ }^{25}$. C'est ainsi que nous cherchons à montrer, en examinant les difficultés que rencontre le processus de l'intervention pour établir une démarche participative dans le cadre d'une opération urbaine, que l'analyse des présupposés qui organisent l'action publique sur ces territoires permet d'illustrer la violence exercée contre certaines catégories de la population martiniquaise.

\footnotetext{
$24 \quad$ Florence Giust-Desprairie, Le Désir de penser, Paris, Téraèdre, 2004, p. 70.

25 Ce risque a été bien perçu par les chercheurs qui prônent une approche en faveur d'un "développement territorial intégré ", faisant de l'intervention auprès des communautés défavorisées « la base d'une stratégie d'intégration des divers sous-systèmes de la société locale ». Pour plus de détails, voir l'article de Juan-Luis Klein, "Innovation sociale et le développement territorial, Introduction ", Revue canadienne des sciences régionales, vol. 32, 2009, p. 13-22.
} 


\section{L'intervention psychosociale clinique en tant que recherche en situation}

Il apparaît ainsi que les différents regards que des disciplines des sciences sociales (géographie, sociologie, économie...) portent sur la réalité sociale de ces sociétés ne peuvent prétendre à une certaine objectivité que si elles sont capables, à travers les méthodes qu'elles mettent en œuvre pour construire leurs objets, d'accorder une attention soutenue au contexte par rapport auquel ces analyses prennent $\operatorname{sen}^{26}$. C'est en tenant compte de ces contraintes, dans le cadre constitué par l'intervention urbaine, que nous avons fait l'hypothèse que les résultats de notre travail de recherche permettaient d'éclairer différemment la question du changement social ${ }^{27}$ dans ces sociétés postcoloniales. Nous considérons, en effet, que l'intervention psychosociale clinique, qui se spécifie d'être une recherche en situation, s'impose, dans l'attention qu'elle accorde aux conditions d'instauration du dispositif sur lequel elle s'appuie pour produire ses résultats, une exigence de rigueur qui n'a rien à envier à d'autres types de démarches scientifiques $^{28}$. Cela se traduit notamment dans le rôle du consultant visant à aider ceux qui ont sollicité son intervention à aborder avec plus de lucidité les problèmes qui les préoccupent, espérant ainsi se donner une chance de participer, avec plus d'efficience, à la situation dont ils sont parties prenantes. Cette visée de développement d'une plus grande autonomie personnelle ou

$\overline{26}$ La critique que Juan-Luis Klein fait des approches visant à promouvoir des actions innovantes dans le champ du développement local permet de voir les lacunes des modèles théoriques et des référents sur lesquels s'appuient ces dispositifs d'intervention pour produire des changements; Juan-Luis Klein, "Territoire et régulation : l'effet structurant de l'initiative locale ", Cahiers de recherche sociologique, $\mathrm{n}^{\circ} 45,2008$, p. 41-57.

27 Nous désignons ainsi un processus initié par le vœu formulé par des hommes et des femmes de parvenir à une citoyenneté pleine et entière susceptible de créer les conditions pour l'amélioration de leur existence et que certains d'entre eux ont cru voir réalisé lorsque la loi hissant la Martinique, en 1946, au statut de département français fut votée.

28 Jacqueline Barus-Michel, "Clinique et sens ", dans Jacqueline Barus-Michel, Eugène Enriquez et André Lévy (dir.), Vocabulaire de psychosociologie, Paris, Érès, 2002. 
d'émancipation sociale, qui soutient la démarche du consultant, est donc susceptible de l'engager à mener un travail de réflexion théorique, en situation, dès lors qu'il a le sentiment que les conditions requises pour l'instauration du dispositif lui font défaut. Car comme l'écrit Florence Giust-Desprairie : « La clinique comme théorie pose la connaissance, non seulement au niveau du contenu, mais dans la prise en compte du contenant. Le dispositif de recherche "parle » et produit par lui-même des significations qui sont à comprendre et orientent l'intelligibilité des contenus ${ }^{29}$ \%. On comprend alors que le premier critère d'appréciation de la validité des résultats de la recherche est celui constitué par la capacité de l'intervention à produire des connaissances théoriques ${ }^{30}$ utiles, non seulement pour le consultant, mais aussi pour ceux avec qui il est en relation.

C'est dans ce sens qu'il convient de voir également dans la démarche du consultant ${ }^{31}$, cherchant à établir le dispositif sur des valeurs et des principes d'ordre démocratique, une forme d'engagement qui, sans pour autant permettre de confondre l'intervention avec une activité politique militante ${ }^{32}$, participe à élargir la portée des résultats de cette recherche en situation bien au-delà

29 Florence Giust-Desprairie, op. cit., p. 52.

30 Cette façon pour nous de concevoir la nature des connaissances produites par le processus de l'intervention prend appui sur ce que nous dit Cornélius Castoriadis au sujet de la théorie. Il nous rappelle, en effet, que la théorie, "c'est la theôria: le regard qui se met en face de quelque chose et l'inspecte"; Cornélius Castoriadis, Figures du pensable, Paris, Seuil, 1999, p. 139. Il apparaît ainsi que la posture que le chercheur choisit d'adopter dans le processus de la recherche a autant d'influence dans la détermination des connaissances produites que le corpus théorique par rapport auquel est adossée la recherche.

31 Pour plus de précisions sur ce qui distingue l'intervention psychosociologique de la pratique d'autres professionnels de la consultation (ingénieurs en organisation, formateurs, psychotechniciens, etc.) ou encore de la rechercheaction d'inspiration lewinienne, nous renvoyons le lecteur à l'article de Jean Dubost et André Lévy paru dans l'ouvrage collectif Vocabulaire de psychosociologie; Jean-Dubost et André Lévy, "Recherche-action et intervention ", dans Jacqueline Barus-Michel, Eugène Enriquez et André Lévy (dir.), Vocabulaire de psychosociologie, Paris, Érès, 2002.

32 Sur la question des rapports de l'intervention psychosociologique avec le politique, on peut consulter l'ouvrage de André Lévy, Sciences cliniques et organisations sociales, Paris, Presses universitaires de France, 1997. 
des limites physiques dans lesquelles s'insère la relation de consultation. Cependant, il ne s'agit jamais pour le consultant de satisfaire ses désirs propres ou inclinations personnelles. "Coopérer au dégagement des facteurs et effets d'aliénation engage le psychosociologue, au-delà du thérapeutique, comme le " psy ", dans la dimension du politique, qui après tout lui est spécifique, pour que puisse se travailler la question du pouvoir ${ }^{33}$ ". Car, comme l'indique Jacqueline Barus-Michel, cette orientation de la recherche reste subordonnée à un travail d'élucidation des conduites.

Les propos de Barus-Michel permettent également de voir que le contexte ne saurait être réduit dans l'analyse à un décor, c'està-dire aux éléments inertes d'une scène sur laquelle prendraient place des personnages. Car ces éléments, dans la mesure où ils apparaissent dotés d'une certaine épaisseur historique et sociale, montrent que le milieu propre des demandeurs, en tant que cadre institutionnel, institue la relation clinique.

Cependant, il convient de ne pas perdre de vue que le contexte n'est pas une donnée sensible qui s'offre d'emblée au regard du consultant. C'est une reconstruction qui repose sur des coordonnées d'espace et de temps en rapport avec l'identité des personnes et des groupes en présence. Cette conception des modalités d'insertion du dispositif d'intervention semble aussi partagée par des chercheurs d'autres disciplines (historiens, géographes...) qui s'intéressent à l'urbain ${ }^{34}$. C'est ainsi qu'une historienne comme Sandra Jathy Pesavento écrit :

Le temps et l'espace sont donc des dimensions pour analyser le phénomène urbain, des référentiels qui possèdent un sens et qui sont liés entre eux : chaque découpage du territoire, chaque forme, chaque matière contient une temporalité qui renvoie à des acteurs, à des pratiques et à des significations. De même, chaque moment du passé laisse des marques objectives dans l'espace ${ }^{35}$.

33 Jacqueline Barus-Michel, op. cit., p. 319.

34 Voir l'ouvrage collectif Hélène Rivière d'Arc et Maurizio Memoli (dir.), Le Pari urbain en Amérique latine. Vivre dans le centre des villes, Paris, Armand Colin, 2006.

35 Sandra Jathy Pesavento, «Ville, espace et temps : réflexions sur le patrimoine urbain au Brésil », dans Hélène Rivière d'Arc et Maurizio Memoli (dir.), Le 
Il ne saurait donc être question de récuser tout recours à la subjectivité arguant du fait que l'objet sur lequel porte l'analyse possède une matérialité qui permettrait de l'appréhender dans sa totalité en faisant abstraction de tout contenu idéel. La nature du phénomène urbain montre, en effet, qu'il s'agit aussi pour le chercheur d'être capable d'élaborer un cadre de références par rapport auquel des conduites et des comportements pourront prendre sens. Ce qui signifie également que le sens dont il est ici question ne saurait être réduit aux seuls éléments susceptibles d'être fournis par des personnes pour expliquer leur comportement.

C'est donc cette conception de l'intervention qui nous a conduit à attribuer à la ville une place centrale dans nos analyses. Car il nous est très vite apparu que cet objet était relié sur le plan de la représentation à un ensemble de significations symboliques et imaginaires que nous ne pouvions pas ne pas intégrer dans l'analyse, au risque d'établir notre démarche sur les principes d'un modèle "managérial ${ }^{36}$ ». C'est ainsi que nous avons été amené à élargir nos investigations bien au-delà des limites de la cité, et à remonter à une époque beaucoup plus ancienne pour comprendre comment des hommes et des femmes avaient été conduits à faire le choix, à un moment de leur vie, de venir s'installer dans des villages de tôles à la périphérie de la ville.

\section{Quelques repères socio-historiques pour situer le contexte de l'intervention urbaine}

En 1848, l'esclavage est aboli dans les Antilles françaises. Cette date marque la fin de la société officiellement structurée selon un mode socio-racial. Elle correspond également à l'époque de mouvements de populations internes à ces régions dans la mesure où les anciens esclaves peuvent alors s'émanciper du cadre de la plantation. Comme l'écrit Massé :

Pari urbain en Amérique latine, op. cit., p. 17.

36 Marie-Hélène Bacqué et al., "La Démocratie participative urbaine face au néo-libéralisme ", op. cit. 
Les années qui suivront l'abolition de l'esclavage seront donc témoins de la constitution d'une véritable paysannerie antillaise, jouissant de leur liberté toute fraîche, les anciens " esclaves casés " fuiront les plantations pour aller $s^{\prime}$ installer sur les terres vierges de leurs îles, dans les parties les plus accidentées impropres à la culture de la canne ${ }^{37}$.

C'est aussi la période où l'on assiste à l'émergence d'une petite bourgeoisie de couleur, implantée majoritairement dans les villes, compte-tenu de la mainmise du groupe des Blancs créoles, propriétaires des grandes exploitations agricoles, sur le patrimoine foncier, situation héritée de l'esclavage qui n'était pas remise en cause par le décret décidant de son abolition.

1946 représente une autre date importante de l'histoire de ces sociétés, puisque c'est à cette date qu'est votée la loi aboutissant à faire sortir la Martinique du statut juridique de colonie, pour accéder à celui de département français d'outre-mer. Cependant, Aimé Césaire qui fut l'un des principaux artisans de ce projet, faisant le bilan de ce processus, soixante ans plus tard, regrettait que celui-ci ait coïncidé avec un effondrement économique du pays $^{38}$.

Les conséquences en furent l'exode : l'exode rural vers les villes du centre de la Martinique ou l'exil vers la France métropolitaine.

C'est ainsi que l'évolution de cette société s'inscrit dans un mouvement d'ensemble qui conduit à la disparition de l'environnement de la société rurale martiniquaise sous l'influence du développement de la société industrielle ${ }^{39}$. Ce phénomène établissant l'évolution de la société martiniquaise sur le modèle de développement des sociétés modernes, celui fondant leur dynamique sur le conflit entre deux stades d'évolution, recèle ses

$37 \quad$ Raymond Massé, La Fin des plantations..., op. cit., p. 29.

38 (Interview de) Aimé Césaire publiée dans le France-Antilles, numéro spécial, avril 2008.

39 Car comme le rappelle Raymond Massé (La Fin des plantations..., op. cit.), déjà pour la période précédant l'abolition de l'esclavage, les liens d'interdépendances entre les économies occidentales et celles des sociétés de plantation impliquent que le développement du système de plantation ne peut être analysé indépendamment du développement du capitalisme marchand en Europe. Dans ce sens, l'essor de l'industrie en France a une répercussion directe sur l'économie martiniquaise à la même époque. 
propres spécificités. La disparition de la société rurale martiniquaise, orchestrée par la loi d'assimilation de $1946^{40}$, l'inscrivant dans un rapport de domination entre centre et périphérie, induit un caractère de facticité dans le développement de cette sociétét ${ }^{41}$; aucune industrie de remplacement n'a vu le jour. Il apparaît ainsi que l'exode rural consécutif à la déstructuration de l'économie coloniale fera de la ville un acteur majeur de l'intégration sociale de la société martiniquaise. L'édification de nombreux bidonvilles à la périphérie de la ville à partir de cette période représente le signe le plus révélateur de ce phénomène. Cependant, cet événement ne constitue qu'un moment fort d'un processus historique faisant, très tôt, de la ville un centre de tensions de tous ordres (économique, social, culturel, politique...).

En effet, la ville (entendons surtout en l'occurrence dans ce terme : la ville portuaire), établie comme le prolongement naturel de l'exploitation agricole esclavagiste, comme lieu de transition de l'exportation vers la métropole de marchandises produites à l'intérieur des terres, va, très rapidement, sous l'influence d'un ensemble de facteurs (ouverture sur l'extérieur, présence d'une population d'hommes de couleur libres, mixité sociale...), s'autonomiser des structures rigides de la société de plantation $^{42}$. Refuge d'une population d'hommes de couleur libres sans cesse croissante, sensible aux valeurs de citoyenneté et d'égalité sociale venues de la métropole, la ville deviendra le lieu fédérateur de dynamiques insurrectionnelles pour des populations aspirant à une amélioration de leur condition (révolte de Saint-Pierre du 21 et 22 mai 1848).

$\overline{40}$ Ce propos prend appui sur la thèse de Raymond Massé qui considère que la départementalisation, en provoquant un changement fondamental des structures de la société martiniquaise, c'est-à-dire en faisant jouer à la Martinique le rôle de marché captif pour la métropole, est à l'origine de la disparition de la paysannerie martiniquaise. Voir Raymond Massé, La Fin des plantations..., op. cit., p. 105.

41 Ce sont de tels faits qui conduisent Édouard Glissant à soutenir, dans son livre, Le Discours antillais, la thèse du " caractère artificiel " et " non fonctionnel " de ce qu'on appelle les classes sociales à la Martinique; Edouard Glissant, Le Discours antillais, Paris, Seuil, 1981, p. 109-111.

42 On peut consulter, à ce sujet, les travaux de Caroline Oudin-Bastide, Travail, capitalisme et société esclavagiste, Paris, La Découverte, 2005. 
Mais, ce que ne saurait masquer ce phénomène, c'est la dimension sociale et culturelle d'un conflit interne à cette société entre deux catégories de population. Il s'agit de celui qui oppose une élite petite bourgeoise très tôt implantée dans la ville ayant épousé, comme le dit Césaire ${ }^{43}$, un certain nombre d'idées héritées du colonialisme et une population rurale plus récente, en quête d'une amélioration de ses conditions de vie ${ }^{44}$. Ces propos de Césaire semblent pouvoir trouver une caution scientifique dans les travaux d'un anthropologue comme Jean Galap qui identifie deux pôles dans l'identité culturelle antillaise : le pôle de la culture créole domestique et celui de la culture française créole. Cette structure bipolaire de l'identité culturelle antillaise, référant à deux systèmes de valeurs distincts, apparaît, sur le plan symbolique, comme le reflet du positionnement des individus et des groupes dans les structures socio-économiques et dans l'espace géographique, traduisant ainsi un rapport dominant/dominé ${ }^{45}$. Dans ce sens, la ville semble avoir symbolisé à différentes périodes et pour différentes catégories de population la possibilité d'un nouveau départ. Ce qui nous permet de retrouver là, ce que Michel Lussault considère être une des fonctions majeures de l'espace géographique : " assurer le régime de visibilité des réalités sociales ${ }^{46} "$.

Cette façon pour nous d'appréhender l'objet d'étude permet de jeter un autre regard sur les opérations de rénovation urbaine. Car il est permis de faire l'hypothèse que les opérations de rénovation urbaine, visant à l'amélioration des conditions de vie dans les quartiers périphériques, s'apparentent, sous un autre aspect, à un processus d'acculturation bouleversant les repères physiques et symboliques sur lesquels sont fondées des identités individuelles et de groupes et sont à l'origine de crises profondes.

43 Aimé Césaire, homme politique et intellectuel engagé, occupa la fonction de maire de la ville de Fort-de-France de 1945 à 2001.

44 (Interview de) Aimé Césaire, France-Antilles, numéro spécial, avril 2008.

45 Jean Galap, "De la famille matrifocale à la famille nucléaire », dans Brigitte Camdessus (dir.), Quand les grands-parents s'en mêlent, Paris, ESF, 1993, p. 60.

46 Michel Lussault, "Bienvenue dans la nouvelle lutte des places! », [en ligne] http://www.raison-publique.fr/article491.html. 
Ce regard permet de dévoiler toute la violence d'une action s'inscrivant dans un rapport entre deux parties dont l'une domine l'autre. L'initiative du projet partant du centre vers la périphérie, il est alors possible de situer l'intervention dans un champ de tension entre deux "pôles d'intensités inégales ${ }^{47}$ ".

\section{Le regard psychosocial clinique}

Cette démarche qui cherche à mieux articuler toutes les dimensions (historiques, sociales, territoriales, économiques et politiques) du champ de l'intervention fait partie intégrante d'une volonté de prendre en compte le caractère pluridimensionnel du contexte d'émergence des phénomènes soumis à l'analyse. Elle se justifie donc par la manière dont la clinique se positionne concernant la question de l'interprétation de la conduite humaine. Elle considère, en effet, que pour son élucidation la prise en compte des coordonnées de la situation, dans laquelle est placé le sujet, fait partie intégrante du processus de construction du sens ${ }^{48}$.

Il apparaît ainsi que le lieu de l'intervention urbaine est celui d'un conflit aux dimensions multiples et que c'est au travers de cette caractéristique-là qu'il convient de penser un objet de cette nature. Pour cela, il nous faut nous départir d'une orientation de pensée qui conduit habituellement à traiter ces objets (la ville, le quartier ou la cité en l'occurrence) " comme sìls désignaient des choses", dans le sens où ils se situeraient dans le réel sur le même plan que les autres éléments du monde physique ${ }^{49}$. Leur dynamique, on le sait, dépend aussi de la façon dont ils sont investis par les individus qui les composent. C'est ainsi que les méthodes et les techniques mises en œuvre par l'intervenant, pour éclairer les acteurs sur les enjeux d'une situation à laquelle ils sont parties prenantes, donnent accès à des phénomènes psychosociaux difficilement appréhendables par d'autres moyens.

\footnotetext{
47 Jean Dubost, Psychologie et intervention, Paris, L'Harmattan, 2006.

48 Daniel Lagache, L'Unité de la psychologie, Paris, Presses universitaires de France, 2004.

49 André Lévy, Sciences cliniques et organisations sociales, Paris, Presses universitaires de France, 1997, p. 27.
} 


\section{L'intervention psychosociologique dans le milieu urbain : entre démarche participative et consultation " palliative"}

La fréquence des interventions, dans le milieu urbain, de professionnels amenés à recourir à des outils et à des techniques des sciences sociales semble, aujourd'hui, s'accélérer en raison de la nécessité pour les responsables politiques de prouver l'utilité des projets qu'ils mettent en place sur leur territoire. À cet effet, des enquêtes sont diligentées auprès des populations dans le but de recueillir les données statistiques et sociales qui serviront à étayer la demande de financement du projet. Cette démarche nécessaire pour un bon calibrage de l'action implique l'information de la population. C'est à cette étape du projet qu'est très souvent cantonné le rôle de l'intervenant. Si bien que cette forme de participation, compte tenu du niveau où elle se situe et des fonctions qu'elle remplit, peut être qualifiée de "palliative".

Par ailleurs, les responsables politiques sont aussi amenés à faire appel à des professionnels pour les aider à mettre en place des dispositifs de concertation pour se conformer aux recommandations des pouvoirs publics incitant les élus locaux à associer l'ensemble des acteurs à la démarche de projet. Mais comme le remarquent Pierre Lefèvre et Michel Sabard ${ }^{50}$ étudiant les évolutions dans le domaine de l'urbanisme, en dépit des efforts fournis par les autorités pour décliner un calendrier participatif à l'usage des aménageurs, cela s'est rarement traduit par une association effective de la population dans les projets urbains; ce processus favorisant davantage la consolidation d'une techno-structure excluant la participation des populations.

La façon dont des urbanistes abordent la question de la participation nous paraît assez révélatrice de la perplexité que l'on peut éprouver face à la formulation de ces demandes d'intervention. Mais ce qui distingue ou au contraire peut contribuer à rapprocher l'attitude du psychosociologue de celle des chercheurs privilégiant une approche en extériorité de ces phénomènes, c'est le fait que son positionnement dans la situation l'oblige à s'enga-

$50 \quad$ Pierre Lefèvre et Michel Sabard, Les Écoquartiers, Rennes, Apogée, 2009. 
ger en faveur de l'une ou de l'autre des deux options qui s'offrent à lui. Car, il apparaît évident que dans ces conditions, à travers les décisions qu'il prend et les options qu'il choisit, il peut soit aider les acteurs à prendre part de façon plus lucide à un projet dans lequel ils sont impliqués, et pour ce faire s'appliquer à réduire la force d'emprise que ceux qui sont à l'initiative de l'intervention peuvent chercher à maintenir sur des personnes ou des groupes et dont les effets ne manquent pas de retentir sur le dispositif technique, ou au contraire contribuer par son positionnement à un processus d'atomisation des collectifs auprès desquels il se situe.

\section{Récit de l'intervention dans la cité $\mathrm{X}$}

L'intervention que nous avons choisie de présenter dans cet article, affichant une volonté de créer le premier écoquartier sur ces territoires semblait offrir, au départ, toutes les conditions favorables permettant de structurer le dispositif technique de façon à en faire le lieu d'un dialogue possible entre les porteurs du projet, la municipalité, et la population du quartier dans lequel devaient être édifiés les nouveaux logements censés les accueillir. Il n'était pas seulement question pour l'intervenant de mener une enquête socio-économique auprès des habitants afin d'éclairer le commanditaire sur la situation, mais aussi de créer les conditions pour asseoir le projet dans un cadre participatif.

Pour répondre aux critères définis dans l'appel d'offres, nous avons structuré notre proposition d'intervention sur un principe d'action conçu pour permettre aux habitants de s'approprier et éventuellement d'amender le contenu du projet d'aménagement proposé.

Outre l'originalité de la démarche, le commanditaire semblait intéressé par les caractéristiques professionnelles de l'équipe d'intervention, composée d'un psychosociologue, d'un psychologue du travail et d'un sociologue. Nous avons interprété cet intérêt pour notre réponse comme la garantie de la prise en compte de la dimension sociale dans un projet urbain, perçu 
comme un véritable challenge sur d'autres plans (technique, économique, politique...).

La cité que la ville souhaitait détruire et reconstruire sous la forme d'un écoquartier était située à un kilomètre du centre ville. Les bâtiments qui la composaient étaient au nombre de trois et comprenaient 318 logements. Ces bâtiments avaient été construits au début des années 1960, afin de reloger des familles délogées d'un village de cases, un peu en contrebas de l'emplacement du site. Les bâtiments avaient mal vieilli et en raison de leur vétusté représentaient, en cas de séisme, un risque majeur pour leurs occupants. Ils étaient occupés par une population assez défavorisée qui, dans la plupart des cas n'avait pas les moyens de se loger ailleurs, alors même qu'un grand nombre de ces occupants étaient propriétaires de leur logement. La ville voulait construire pour les remplacer 500 logements de grand standing dont l'impact sur l'environnement serait très limité. Pour cela, elle avait fait l'acquisition d'un ensemble d'autres parcelles limitrophes. C'est ainsi que le nouvel ensemble était prévu pour s'établir sur une superficie de 4,5 hectares. Le commanditaire estimait alors qu'il n'était plus concevable de garder l'appellation de cité pour désigner le lieu d'emplacement des nouvelles constructions, aussi cherchait-il déjà à imposer dans les discours la notion de quartier.

Dans son approche de la population habitant sur les lieux, le commanditaire établissait une distinction entre propriétairesoccupants et propriétaires non-occupants. Ces derniers nous étaient présentés sous un jour plutôt défavorables, puisqu'ils étaient considérés dans leur grande majorité comme des " marchands de sommeil ", c'est-à-dire comme des personnes qui avaient fait l'acquisition de ces logements, qualifiés d'insalubres, dans le but de les louer, moyennant un loyer prohibitif. Le commanditaire s'attendait à ce que ces personnes se montrent les plus résistantes à l'idée de céder leur logement à la ville au prix fixé par la loi en matière d'expropriation; et cela, d'autant plus qu'il n'était pas question d'étendre à cette catégorie de propriétaires le dispositif d'aide qui leur aurait permis de faire l'acquisition d'un logement, dans l'ensemble qui serait nouvellement 
construit, dans les conditions prévues pour les propriétairesoccupants. Par contre, la ville s'occuperait du relogement des locataires des propriétaires non-occupants. Une fois indemnisés, les propriétaires non-occupants qui souhaiteraient faire l'acquisition d'un logement dans le quartier reconstruit seraient contraints de le faire au prix du marché.

Pour les propriétaires-occupants, le sujet d'inquiétude n'était pas le même; ce que le commanditaire craignait le plus, c'était le rôle que pourrait jouer auprès d'eux un opposant politique. La crainte était qu'il profite des frustrations et des angoisses que suscitait l'opération pour capitaliser des soutiens dans sa lutte politique contre la majorité en place au niveau de la municipalité.

Toutes ces appréhensions n'étaient pas seulement manifestes sur le plan du discours à l'adresse des consultants. Elles se traduisaient dans des actes qui paralysaient le démarrage sur le terrain de l'action. Si bien que pour les dépasser, nous avons dû nousmêmes prendre l'initiative, une fois notre lettre de mission signée, de provoquer une rencontre avec la population afin de l'informer sur notre rôle dans l'opération. Cette première rencontre fut assez houleuse, beaucoup redoutaient la mise en œuvre de ce projet. Deux clans semblaient s'opposer quant à l'attitude à adopter. Chez les propriétaires-occupants, un comité de défense du quartier s'était formé alors que les propriétaires non-occupants, s'ils se montraient particulièrement hostiles au projet, étaient surtout représentés par de fortes individualités qui ne parlaient pas au nom d'un groupe organisé. Les propriétaires-occupants, davantage préoccupés par le montant de l'indemnité qui leur serait allouée pour la reprise de leur logement, étaient partisans de laisser démarrer le projet. Par contre les propriétaires nonoccupants refusaient toute collaboration dans le projet, nous signifiant ainsi que nous n'étions pas les bienvenus dans le quartier. Une représentation, bien partagée, circulait parmi les participants à cette réunion, elle reposait sur l'idée que ce projet de rénovation du quartier servirait à masquer un autre projet moins avouable : celui d'une opération de spéculation immobilière dont ils seraient les victimes désignées. C'est ainsi que notre 
rôle dans ce débat a consisté à faire ressortir la part de fantasme que contenaient ces discours. Et, il aura fallu, au final, une prise de position ferme, en faveur du projet, du comité de défense du quartier, ouvrant ainsi une brèche dans ce front du refus, pour que les propriétaires non-occupants acceptent de participer à l'étude pré-opérationnelle qui nous était confiée.

À partir du moment où nous avons eu le sentiment d'avoir été autorisés à rester dans le quartier par les personnes auprès desquelles nous étions censés intervenir, nous avons commencé notre travail d'investigation. C'est ainsi que nous avons mené une enquête par questionnaire auprès des occupants de 210 logements. Le travail d'enquête en porte-à-porte s'est étalé sur une durée de 6 mois. Les données recueillies ont été traitées à l'aide du logiciel de traitement d'enquête Sphinx.

Ce travail d'enquête fut ensuite complété par des entretiens en face-à-face dans la permanence que nous tenions deux fois par semaine dans le quartier. Au final, ce sont les occupants de 249 logements avec lesquels nous avons pu nous entretenir. Ces échanges nous ont permis de disposer d'éléments issus d'une histoire passée que nous pouvions mettre en rapport, avec ce que nous livrait l'analyse de parcours et de trajectoires d'individus et de groupes, pour comprendre et aider des personnes qui s'adressaient à nous à se projeter dans l'avenir, du moins mieux qu'elles ne pouvaient le faire, jusqu'à présent, traversées qu'elles étaient par des moments de doute, d'angoisse, mais aussi d'espoir, pas toujours fondés, faute de s'appuyer sur une appréhension claire de ce qui constituait leur situation.

C'est ainsi que nous pouvions avec certains de nos interlocuteurs remonter à une époque antérieure à celle de la construction des bâtiments, au cours des années 1960, période où ils vivaient encore dans leur quartier de cases. Il s'agissait d'un bidonville qu'ils avaient habité enfants ou jeunes couples, dans des cases, insalubres, construites sur des parcelles de terre sur lesquelles ils s'étaient installés. L'entassement des cases était tel qu'on ne pouvait circuler dans le quartier qu'à travers un dédalle de ruelles étroites et mal entretenues. L'un de mes interlocuteurs me 
montrant une cicatrice sur son corps, comme preuve irréfutable de la véracité de ses dires, témoignait ainsi de la fréquence des bagarres dans ce quartier dans lequel la police ne pénétrait pas. Ils en furent délogés dans les années 1960 pour être relogés dans ces bâtiments nouvellement construits. À l'époque, l'architecture des bâtiments en faisait un lieu d'habitation dont ils étaient fiers. C'est à partir des années 1980, lorsque les bailleurs sociaux ont commencé à céder les logements à ceux qui y habitaient que les choses se sont dégradées ${ }^{51}$. Cependant, tous les locataires de l'époque n'ont pas opéré un choix identique, certains restant, jusqu'à présent, locataires d'un logement pour lequel ils s'acquittent d'un loyer modique.

La trame de cette histoire n'était plus la même lorsque l'interlocuteur était arrivé dans le quartier au cours des années 1980, c'est-à-dire lors de la période où les bailleurs sociaux ont décidé de proposer des lots à l'achat. Ce sur quoi semblait être organisée la trame de ces discours, était l'idée que la mise en vente de ces lots, à un prix très abordable, représentait une " bonne affaire " à saisir. Il s'agissait d'abord pour ces personnes de réaliser un placement financier. Ce type de discours se distinguait donc très nettement de celui des propriétaires non-occupants qui avaient réellement vécu dans la cité. Ces derniers, bien que n’habitant plus la cité, n'avaient nullement l'intention de céder leur appartement à la municipalité. Pour eux, ce bien représentait un patrimoine qu'ils souhaitaient transmettre à leurs enfants.

Donc, au fur et à mesure que nous progressions dans la compréhension de la situation, nous pouvions mieux cerner la part de méconnaissance que contenaient ces discours qui tendaient à faire de l'ensemble des propriétaires non-occupants des " marchands de sommeil ». Cette représentation partagée au niveau de la municipalité semblait constituer un obstacle majeur sur le plan de la communication, car elle ne permettait pas de prendre en compte l'ampleur des investissements, tant sur le plan

51 Nous verrons, plus loin, que cette situation trouvait son explication dans la difficulté pour les habitants de constituer un syndic, dans un ensemble comptant, désormais, des propriétaires et des locataires logés par les bailleurs sociaux. 
affectif que matériel, qu'avaient pu consentir des personnes, parties de " rien " (les terrains sur lesquels elles avaient construit leur première habitation ne leur appartenant pas), pour améliorer leur condition. Autrement, on aurait pu concevoir que le projet de les exproprier les renvoyait au souvenir d'un passé douloureux qu'ils croyaient définitivement révolu, dès lors qu'ils étaient devenus propriétaires d'un bien immobilier. Cette interprétation pouvant être étendue à l'analyse du positionnement des propriétaires-occupants dans la mesure où eux aussi prenaient conscience que leurs moyens actuels ne leur permettraient pas nécessairement d'accéder à un statut de propriétaire dans le nouveau projet de construction.

Sur ce fondement, il n'était pas non plus permis d'écarter l'idée que ce projet, tel qu’il était présenté, venait accentuer un clivage plus profond : celui né au tournant des années 1980, lorsque les bailleurs sociaux avaient décidé de se retirer de la gestion de ce parc immobilier. Car on peut faire l'hypothèse que jusqu'à cette période ces personnes pouvaient encore s'identifier entre elles à l'image d'une communauté soudée. Cette représentation imaginaire du groupe prendrait ses racines, sur le plan de la réalité, dans le souvenir de luttes et d'affrontements menés par des personnes et des groupes pour se maintenir sur des terrains qu'ils s'étaient alors appropriés pour fonder une famille et mener une existence collective. C'est ainsi que la décision prise dans les années 1980 peut être appréhendée comme introduisant une rupture sur le plan imaginaire dans la façon pour ces personnes de se percevoir sous une identité collective; elle va être à l'origine d'un processus de morcellement de cette communauté, la partageant dès lors en un ensemble de groupes distincts.

Nous avons trouvé les indices de ce clivage dans le discours que des propriétaires-occupants pouvaient tenir sur les locataires qui n'avaient pas fait le choix de devenir propriétaires. Ils les voyaient comme des "profiteurs", des gens qui veulent " tout sans rien donner en contrepartie ".

L'un des effets plus concret de ce clivage se traduira dans l'impossibilité pour les habitants de constituer un syndic, après 
la procédure de cession de lots engagée en 1980 par les bailleurs sociaux. Cette difficulté sera à l'origine d'un processus conduisant à l'absence d'entretien des parties communes et à la dégradation des bâtiments. Ce processus, s'accentuant au fil du temps, accompagnera le départ d'un nombre sans cesse croissant de propriétaires-occupants; ceux dont la situation matérielle s'était améliorée, entre-temps, et qui pouvaient faire l'acquisition d'une seconde résidence.

Dans ce sens, cette analyse de la situation permet de saisir, dans ses grandes lignes, un processus de hiérarchisation sociale en lien avec des étapes marquantes de la vie de cette communauté. Mais, s'il paraît difficile de situer l'amorce de ce processus de façon précise d'un point de vue historique, ainsi que de définir son ampleur sur le plan spatial, nous pouvons néanmoins considérer que certaines de ses caractéristiques formelles pouvaient induire dans les esprits une analyse en résonance avec la notion de « classe sociale ${ }^{52} »$.

C'est à ce schéma de pensée que l'on pourrait attribuer l'ancrage d'une représentation des propriétaires non-occupants comme des " personnes cupides " ou comme des " rentiers " seulement préoccupés par les profits qu'ils pouvaient tirer de la location de leurs biens. C'est à ce même schéma que l'on pourrait aussi attribuer la part de fascination qu'exerçait chez les personnes l'édification d'un quartier huppé sur le site. Aux uns, il inspirait un discours et des sentiments de rancour et de frustration contre la municipalité et la clique des "gros bonnets " qui voulaient profiter de l'occasion pour mettre en place une opération de spéculation immobilière, dont eux les "petits ", les "sans-grade » feraient les frais; et aux autres, la possibilité, en recevant une

52 Ce terme fait référence à la façon dont des personnes, en utilisant les moyens qu'elles avaient à leur disposition, essayaient de donner du sens à la situation, et, ce faisant, essayaient de permettre à d'autres de pouvoir aussi s'y reconnaître. Car, comme le montre la suite du texte, les notions qu'elles pouvaient utiliser pour accréditer l'idée de l'existence d'un collectif, d'un " nous ", ne semblaient pas tenir compte de la diversité des intérêts particuliers. C'est ainsi que ces significations ne paraissaient pouvoir prendre sens qu'à l'intérieur du discours qui servait à les véhiculer et donc se placer ainsi au service de stratégies exprimant d'abord la singularité d'un « je». 
indemnisation conséquente pour leur logement, de faire partie de ces privilégiés, d'inverser la hiérarchie sociale qui s'était établie dans les années 1980, lorsque des propriétaires avaient pris la décision de quitter le quartier et de louer l'appartement qu'ils occupaient.

C'est ainsi que tout semblait opposer ces deux catégories de propriétaires. Si bien qu'il était inenvisageable qu'ils puissent se regrouper au sein d'une même structure pour défendre leurs intérêts.

Concernant le commanditaire, il nous est apparu que le discours qui faisait des propriétaires non-occupants un groupe de rentiers servait, en grande partie, à masquer la part de mauvaise conscience que l'on pouvait avoir à l'égard de ces personnes que l'on projetait d'exproprier. Car, lorsque l'on prenait en compte l'histoire de ces personnes, de modestes ouvriers, qui avaient dû pour se loger dans la ville, habiter des cases de tôles, il devenait difficile de voir en elles, par la suite, des rentiers, même si l'on pouvait, à cet effet, invoquer la réussite du parcours qui leur avait permis de quitter la cité. Donc ce raccourci sur le plan de la pensée visait d'abord pour le commanditaire à se dégager d'un ensemble d'obligations envers ces personnes, afin de faire face à d'autres contraintes sur le plan de la réalisation du projet.

L'une des principales contraintes à laquelle devait faire face le commanditaire se situait sur le plan du financement du projet. Les fonds publics des institutions nationales et locales ne constituaient pas la totalité du financement du projet. Des acteurs du secteur privé étaient associés au projet, recevant en contrepartie la concession de la gestion d'un ensemble de lots. D'où l'obligation pour le commanditaire de réduire dans les mêmes proportions la part dédiée au secteur aidé, sous peine de grever le budget de l'opération.

Au regard des résultats issus de l'enquête par questionnaires que nous avons réalisée auprès de 210 ménages sur le total des 318 logements que comptait la cité, il apparaissait clairement que la très grande majorité des habitants de la cité (518 personnes recensées par les moyens de l'enquête de terrain) pouvait être 
considérée comme une population précarisée (seulement $13 \%$ des ménages déclaraient être imposables sur le revenu). Dans la cité, le taux de chômage se situait autour de $40 \%$ et la population des personnes âgées était également très élevée $57 \%$ des propriétaires-occupants étaient âgés de 66 ans et plus). Ces chiffres montraient qu'un grand nombre de propriétaires-occupants ne pourraient pas accéder à un statut de propriétaire dans le nouvel ensemble.

C'est donc à travers ce cadre d'analyse remanié que l'on pouvait réinterpréter le sens de la demande d'intervention. Car à mesure que nous progressions dans le processus même de l'intervention, nous avons vu se creuser, au sein du dispositif, un fossé entre les parties. Cela se traduisait par un cloisonnement entre le travail sur le terrain, c'est-à-dire avec la population, et le travail du groupe des intervenants responsables de la conduite du projet sur le plan technique et financier. Nous n'étions pas conviés aux réunions de ce dernier groupe d'intervenants. Et, l'association de la population au projet se faisait dans le cadre de l'organisation de réunions publiques sur le mode d'une communication descendante, l'objectif visé étant essentiellement d'informer les participants sur les dimensions techniques du projet et sur le planning des prochaines étapes.

Ainsi, il devenait évident que cette orientation que l'on cherchait à maintenir au niveau du dispositif de l'intervention visait à préserver la confidentialité des échanges de façon à prévenir les réactions trop hostiles au projet que l'on anticipait d'une communication plus ouverte. Dans cette perspective, le commanditaire n'imaginait pas que les personnes visées par le projet puissent se situer autrement que comme des obstacles au bon déroulement des opérations.

\section{Conclusion}

Dans cet article, nous avons cherché à montrer comment l'attention portée aux modalités d'insertion du dispositif technique d'intervention dans le milieu urbain permet de faire émerger des connaissances théoriques nouvelles, utiles pour la compréhension 
de la dynamique de ces sociétés. Nous avons vu que les stratégies que pouvaient mettre en place les personnes obéissaient davantage à des critères en rapport avec des valeurs et des normes en correspondance avec celles des sociétés modernes (le travail, la propriété, la famille...). C'est ainsi que les divisions et les conflits qu'elles conditionnaient dans les rapports et les relations pouvaient être déchiffrés et traduits en termes de "lutte des places ${ }^{53}$ ", dans la mesure où les difficultés que ces personnes rencontraient pour défendre leurs intérêts n'aboutissaient pas à donner naissance à une conscience collective en référence à des réalités sociales vécues comme traduisant une identité de condition.

Le processus de rénovation urbaine paraissait alors accompagner et voire même favoriser l'éclatement de ce qui aurait pu, en rapport avec l'histoire de cette communauté, la faire apparaître comme un tout, comme un système social relativement autonome. L'intervention révélait, en effet, que l'organisation de cette communauté n'avait pas résisté à la domination écrasante du cadre institutionnel établi. Ses structures, fondées au départ sur l'occupation illégale de parcelles de terrain, auraient pu laisser croire qu'elles s'instituaient à l'encontre du régime légal (la location, la propriété); mais trop labiles, car soumises à des sollicitations institutionnelles incessantes, elles n'ont jamais pu paraître capables d'offrir des garanties suffisantes pour une intégration sociale réussie. Tout comme J. Dubost, intervenant dans une zone rurale des Alpes, nous avons pu constater que la loi d'expropriation qui ne prenait en considération que les titulaires d'un titre de location ou de propriété ne faisait que parachever un processus d'effritement de cette communauté amorcé bien avant le démarrage du projet. Dans sa déclinaison, la démarche établissant une distinction entre propriétaires-occupants et propriétaires non-occupants, figurant, sur le plan de la représentation, l'application d'un principe de justice sociale, semblait alors offrir un cadre suffisant pour contenir les sentiments d'indignation et de frustration. Les groupements ou les tentatives de regroupement auxquels le processus pouvait donner

53 Michel Lussault, op. cit. 
lieu ne paraissaient pas suffisamment forts ou trop éphémères pour peser de façon significative dans le rapport de forces entre ces deux pôles.

Il apparaît en effet que seule la prise en compte du problème de l'inégalité de la répartition du patrimoine foncier entre les différents groupes sociaux constitutifs de la société martiniquaise, situation héritée de la période esclavagiste et de la colonisation, aurait pu permettre aux habitants de la cité de fonder leur engagement sur un principe de justice sociale transcendant les clivages entretenus par la loi d'expropriation. Une telle démarche impliquerait nécessairement une politisation de la situation, réintroduisant des dimensions de l'identité ethnique ou culturelle qui jusque-là n'étaient pas prises en considération dans l'analyse. C'est ainsi que sur un plan plus général, il est possible de considérer l'analyse de cette intervention comme l'illustration d'un processus de dépolitisation de la question sociale dans ces sociétés; privant ainsi des individus et des groupes des moyens de résistance collective qui leur aurait permis de réagir à la situation de violence qui leur était faite. Et, nous avons cherché à montrer que l'on pouvait relier cette situation à un phénomène de forclusion d'un ensemble d'éléments constitutifs de l'identité culturelle antillaise, sous l'influence d'une conception rigide et autoritaire de l'appartenance nationale relayée par une élite de couleur impliquée dans un processus de domination sociale, conduisant ainsi les acteurs à adopter un discours en accord avec celui du régime de l'institution et donc un positionnement dans l'espace public ne permettant pas un " exercice plein de la citoyenneté ». Cette situation contribuant à former des zones " où se condensent les traits que dessinent la face d'ombre d'une formation sociale : la pauvreté, la violence, le racisme, l'insécurité civile et sociale ${ }^{54}$ ".

C'est pourquoi, nous estimons, dans le contexte d'un monde de plus en plus ouvert où les flux migratoires se font sans cesse plus intenses, amenant des hommes et des femmes de couleurs et de cultures différentes à coexister au sein d'un même ensemble

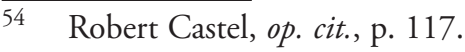


national, que les résultats des recherches conduites dans le cadre des sociétés postcoloniales sont susceptibles d'intéresser bien au-delà des limites de leurs frontières.

\section{Bibliographie}

Bacqué, Marie-Hélène et al., « La Démocratie participative urbaine face au néo-libéralisme ", Mouvements, nos 39-40, 2005, p. 121-131.

Barus-Michel, Jacqueline, "Clinique et sens ", dans Barus-Michel, Jacqueline, Eugène Enriquez et André Lévy (dir.), Vocabulaire de psychosociologie, Paris, Érès, 2002, p. 313-323.

Bastide, Roger, Les Amériques noires, Paris, L'Harmattan,1996.

Blatrix, Cécile, "La Démocratie participative en représentation ", Sociétés contemporaines, $\mathrm{n}^{\circ}$ 74, 2009, p. 97-119.

Blondiaux, Loïc, Le Nouvel Esprit de la démocratie, Paris, Seuil et La République des Idées, 2008.

Blondiaux, Loïc et Yves Sintomer, "L'Impératif délibératif ", Rue Descartes, vol. $1, n^{\circ} 63,2009$, p. 28-38.

Castel, Robert, La Discrimination négative. Citoyens ou indigènes?, Paris, Seuil, 2007.

Castoriadis, Cornélius, Figures du pensable, Paris, Seuil, 1999.

Césaire, Aimé, interview de, France-Antilles, numéro spécial, avril 2008.

Chamoiseau, Patrick, Texaco, Paris, Gallimard, 1992.

Chivallon, Christine, Espace et identité à la Martinique, Paysannerie des mornes et reconquête collective 1840-1960, Paris, CNRS, 2002.

Chivallon, Christine, La Diaspora noire des Amériques, expériences et théories à partir de la Caraïbe, Paris, CNRS, 2004.

Cuche, Denys, «Les Amériques noires dans l'anthropologie et la sociologie françaises depuis Les Amériques noires de Roger Bastide (1967)", Bastidiana, $\mathrm{n}^{\text {os }} 13-14,1996$, p. 119-142.

Dubost, Jean et André Lévy, "Recherche-action et intervention ", dans Barus-Michel, Jacqueline, Enriquez Eugène et André Lévy (dir.), Vocabulaire de psychosociologie, Paris, Érès, 2002, p. 391-416.

Dubost, Jean, Psychosociologie et intervention, Paris, L'Harmattan, 2006. 
Galap, Jean, « De la famille matrifocale à la famille nucléaire », dans Brigitte Camdessus (dir.), Quand les grands-parents s'en mêlent, Paris, ESF, 1993, p. 55-69.

Gilroy, Paul, L'Atlantique noir, Modernité et double conscience, Cahors, Editions Kargo, 2003.

Giust-Desprairie, Florence, Le Désir de penser, Paris, Téraèdre, 2004.

Glissant, Edouard, Le Discours antillais, Paris, Seuil, 1981.

Gret, Marion et Yves Sintomer, Porto Alegre, l'espoir d'une autre démocratie, Paris, La Découverte, 2002.

Hélin, Jean-Claude, "La Concertation de l'article L. 300-2 du code de l'urbanisme ", Paris, Les cabiers du GRIDAUH, 2006, p. 207-224.

Hess, Rémi, Centre et périphérie, Paris, Anthropos, 2001.

Jatahy Pasavento, Sandra, «Ville, espace et temps : réflexions sur le patrimoine urbain au Brésil ", dans Rivière d'Arc et Memoli Maurizio (dir.), Le Pari urbain en Amérique latine. Vivre dans le centre des villes, Paris, Armand Colin, 2006, p. 17-26.

Klein, Juan-Luis, "Innovation sociale et le développement territorial, Introduction ", Revue canadienne des sciences régionale, vol. $32, \mathrm{n}^{\circ} 2$, 2009, p. 13-22.

Klein, Juan-Luis, «Territoire et régulation : l'effet instituant de l'initiative locale ", Cahiers de recherche sociologique, n 45, 2008, p. 41-57.

Lagache, Daniel, L'Unité de la psychologie, Paris, Presses universitaires de France, 2004.

Lefevre, Pierre et Michel Sabard, Les Écoquartiers, Rennes, Apogée, 2009.

Lévy, André, "Les objets introuvables de l'analyse psychosociologique ", Revue internationale de psychosociologie, $\mathrm{n}^{\circ}$ 1, Paris, Eska, 1994, p. 17-26.

Lévy, André, Sciences cliniques et organisations sociales, Paris, Presses universitaires de France, 1997.

Lussault, Michel, «Bienvenue dans la nouvelle lutte des places! », [en ligne] http://www.raison-publique.fr/article491.htlm, consulté le 5 février 2012.

Martouzet, Denis, « Le rapport affectif à la ville, conséquences urbaines et spatiales. Le cas de Fort-de-France / Urban and Spatial Consequences of Affective relation to the City. The Case of Fort-de-France ", Annales de Géographie, vol. 111, n 623, 2002, p. 73-85.

Massé, Raymond, La Fin des plantations? Evolution des formes de soumission du travail dans deux sociétés néo-coloniales: Martinique et Guadeloupe, Montréal, Publication du Centre de recherches Caraïbes, Université 
de Montréal, 1980.

Massé, Raymond, Détresse créole, Ethnoépidémiologie de la détresse psychique à la Martinique, Québec, PUL, 2008.

Nonjon, Magali, «lES Professionnels de la participation : savoir gérer son image militante ", Politix, n 70, 2005, p. 89-112.

Oudin-Bastide, Caroline, Travail, capitalisme et société esclavagiste, Paris, La Découverte, 2005.

Rigouste, Mathieu, L'Ennemi intérieur, la généalogie coloniale et militaire de l'ordre sécuritaire dans la France contemporaine, Paris, La Découverte, 2009.

Rivière d'Arc, Hélène et Maurizio Memoli (dir.), Le Pari urbain en Amérique latine. Vivre dans le centre des villes, Paris, Armand Colin, 2006.

Savidan, Patrick, "Démocratie participative et conflit ", Revue de métaphysique et de morale, $\mathrm{n}^{\circ}$ 58, 2008, p. 177-189.

Sméralda-Amon, Juliette, La Racisation des relations intergroupes ou la problématique de la couleur, Paris, L'Harmattan, 2002. 